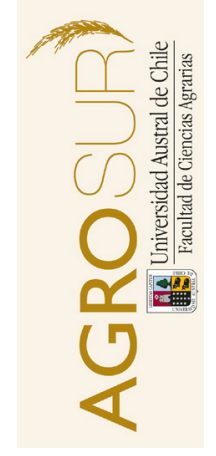

\section{Efecto de la aplicación de lodo de salmón en la calidad estructural de un Ultisol}

\author{
Effect of the application of salmon sludge \\ on the structural quality of an Ultisol
}

\author{
Antipa, Z. ${ }^{a}$, Nissen, J. ${ }^{a}$, Seguel, 0. ${ }^{b, c}$, Clunes, J. ${ }^{a}$, Dörner, J. ${ }^{a, b *}$ \\ ${ }^{a}$ Instituto de Ingeniería Agraria y Suelos, Universidad Austral de Chile. Casilla 567, Valdivia, Chile. \\ ${ }^{b}$ Centro de Investigación en Suelos Volcánicos, Universidad Austral de Chile. Casilla 567, Valdivia, Chile. \\ ${ }^{c}$ Departamento de Ingeniería y Suelos, Facultad de Ciencias Agronómicas, Universidad de Chile, \\ casilla 1004, Santiago, Chile.
}

\begin{tabular}{l} 
A R T I C L E I N F O \\
\hline Keywords: \\
Salmon sludge \\
Structural stability \\
Air permeability \\
\hline Original Research Article, \\
Soil Science \\
\hline *Corresponding author: \\
Zahira Antipa, José Dörner \\
E-mail address: \\
josedorner@uach.cl
\end{tabular}

\begin{abstract}
A B S T R A C T
The application of salmonicola sludge in agriculture allow to improve soil fertility, due to its high content of organic matter and nutrients available. However, there is no clarity about the effect of sludge application on soil physical properties. The objective of this investigation was to determine the effect of the application of salmon sludge on quantitative parameters and functional aspects of the porous system of a soil. Disturbed and undisturbed soil samples were collected from Los Ulmos Series (Typic Paleudults) and different doses of sludge were applied (0, 40 and $\left.80 \mathrm{Mg} \mathrm{ha}^{-1}\right)$. Six wetting and drying cycles were performed with samples assembled with the different sludge applications. In each cycle the air permeability $(\mathrm{Ka})$ and the volumetric changes of the samples were measured. Subsequently, the contact angle and the stability of aggregates were determined. High doses of sludge applied to the soil generate a substitution of macroporosity for pores of water that can be used by plants, decreasing the wCP (or air capacity) by $46 \%$ and increasing the PAW by $14 \%$, which produces an increase in structural stability and resistance to wetting. The air permeability was not affected by the dose of sludge. The recycling of salmon sludge is presented as a viable alternative to be used on degraded soils, but it is undoubtedly necessary to carry out further studies to evaluate the effect of successive doses on the soil regarding e.g. the increase in water repellency.
\end{abstract}

\title{
RESUMEN
}

La aplicación de lodos salmonícolas en la agricultura permite mejorar la fertilidad del suelo, debido a su alto contenido de materia orgánica y nutrientes disponibles. Sin embargo, no existe claridad sobre el efecto de la aplicación de lodos en las propiedades físicas del suelo. El objetivo de esta investigación fue determinar el efecto de la aplicación de lodos de salmón sobre parámetros cuantitativos y aspectos funcionales del sistema poroso de un suelo. Se recolectaron muestras disturbadas y no disturbadas de suelo de la Serie Los Ulmos (Typic Paleudults) y se aplicaron distintas dosis de lodo (testigo o nivel 0, 40 y $\left.80 \mathrm{Mg} \mathrm{ha}^{-1}\right)$. Se realizaron seis ciclos de humectación y secado $(0-60 \mathrm{hPa})$ con muestras ensambladas con las diferentes aplicaciones de lodo. En cada ciclo se midió la permeabilidad de aire $(K a)$ y los cambios volumétricos de las muestras. Posteriormente, el ángulo de contacto y la estabilidad de agregados. Dosis de elevadas de lodo (80 $\mathrm{Mg}^{-1}$ ) generan una sustitución de la macroporosidad por poros de agua aprovechable por las plantas, disminuyendo los PDR (o capacidad de aire) en un $46 \%$ e incrementando los PAU en un 14\%, lo que produce un aumento en la estabilidad estructural y la resistencia a la humectación. La permeabilidad de aire no se vio afectada por la dosis de lodo. El reciclaje de lodo de salmón se presenta como una alternativa viable de ser realizada en suelos degradados, pero sin duda es necesario realizar mayores estudios para evaluar el efecto de dosis sucesivas en el suelo p.ej. en relación al incremento de la repelencia al agua.

Palabras clave: lodos de salmón, estabilidad estructural, permeabilidad de aire.

\section{INTRODUCCIÓN}

La contaminación orgánica durante la producción de salmones en Chile puede llegar a aguas prístinas, cambiar la microfauna bentónica y la química de los sedimentos bajo las jaulas, aumentar riesgos de propagación de enfermedades y por ende, arruinar medios de subsistencia de otros rubros productivos asociados a medios costeros (Celis y Sandoval, 2010). El principal desecho producido por la industria salmoacuícola es el lodo de salmón, material orgánico constituido por heces de peces y restos de alimentos no consumidos (Teuber et al., 2005). Estos desechos incrementan la cantidad de nutrientes en el sistema, tales como fós- 
foro y nitrógeno, favoreciendo la eutroficación de los ecosistemas acuáticos (Mena, 1997). La re-utilización de lodos como fertilizante en la agricultura, principalmente por la cantidad de nutrientes y materia orgánica que presentan, puede reducir la contaminación de los sistemas acuícolas (Ahmed et al., 2010). Lo anterior, puede estar orientado a mejorar suelos que han sufrido procesos de degradación, quedando expuestos a la erosión hídrica y eólica debido a una reducción de la cobertura vegetal (Peralta, 1995). En ese contexto, la recuperación de áreas afectadas por degradación presenta inconvenientes para el establecimiento de especies durante la restauración ecológica, ya que el bajo contenido de materia orgánica y de nutrientes, y la mala calidad estructural afectan negativamente la fertilidad natural y la capacidad de retención de agua (Varnero, 2001). Dado que los lodos obtenidos de las plantas de tratamiento de agua residuales presentan un alto contenido de materia orgánica, estos pueden ser una alternativa de utilización para recuperar suelos degradados (Celis et al., 2008). Además, se ha demostrado que las aplicaciones de lodo generan un aumento en la estabilidad de los agregados. Aportes hasta un 5\% (base masa seca) de lodos urbanos generan aumentos de hasta un $78 \%$ de la estabilidad de agregados respecto a la condición inicial de un suelo ante la erosión (Guerrero et al., 2001).

Los efectos de la aplicación de lodos urbanos son predominantemente físicos, mientras que los efectos químicos y nutricionales dependerán de la composición, tratamiento y método de aplicación (Cuevas et al., 2006; Salazar et al., 2012). Sin embargo, mejorar la fertilidad del suelo es más rápido e inmediato de visualizar que el efecto de los lodos sobre la estructura del suelo. Pinochet et al. (2001), afirman que los desechos sólidos provenientes de la salmonicultura presentan un alto contenido de fósforo, otorgándoles utilidad como abono orgánico fosforado. Por otra parte, los lodos pueden presentar problemas por generar aumentos de la carga metálica de los suelos, especialmente de zinc y cobre, cuando son aplicados con dosis sobre $150 \mathrm{Mg} \mathrm{ha}^{-1}$ (Celis et al., 2006; Aravena et al., 2007).

Durante la formación de los agregados del suelo a través de ciclos de mojado y secado, se produce un reordenamiento de las partículas orgánicas y minerales, generando estructura, lo que depende de la frecuencia y la intensidad de los procesos de hinchamiento y contracción, de la estabilidad mecánica y la presencia de componentes orgánicos, afectando así la resistencia de los agregados (Hallet et al., 2001; Horn, 2002). La proporción de las fases sólida, líquida y gaseosa del suelo cambia continuamente y está afectada por el clima, la vegetación y el manejo del suelo (Hillel, 1998). La MO del suelo es considerada en la mantención de la estabilidad estructural en un amplio rango de tipos de suelos, ya que actúa como un agente cementante de las partí- culas minerales, aumentando la resistencia mecánica y la estabilidad del sistema poroso (Hartge y Horn, 2009; Baldock y Nelson, 1999).

Si bien es cierto que existen antecedentes respecto de la incorporación de MO sobre la estructura y sistema poroso del suelo (Diacono y Montemurro, 2011), no hay mayor información respecto del efecto de los lodos de salmón sobre dichos parámetros, los que podrían ser usados en zonas de Chile en donde se produce este tipo de residuo. En ese contexto, cabe destacar que la Región de Los Ríos presenta en la Cordillera de la Costa suelos denominados Ultisoles, que por su naturaleza y características son más frágiles y, por ende, susceptibles a procesos de degradación que los Andisoles, ya que presentan menores contenidos de materia orgánica, elevadas pendientes y texturas finas que dificultan la infiltración y permeabilidad al agua y al aire (Dörner et al., 2011). Por lo expuesto, el objetivo de esta investigación fue determinar el efecto de la aplicación de distintas dosis de lodos de salmón (testigo o nivel 0, 40 y $80 \mathrm{Mg} \mathrm{ha}^{-1}$ ) sobre las propiedades físicas del sistema poroso de un suelo después de someterlo a sucesivos ciclos de humectación y secado.

\section{MATERIAL Y MÉTODO}

\section{Suelos y lodos}

Se trabajó con un suelo rojo arcilloso de la Serie Los Ulmos (Typic Paleudults), el cual era utilizado bajo pradera naturalizada. Este suelo, que se originó a partir de cenizas volcánicas pleistocénicas, está ubicado en la Cordillera de la Costa ( $40^{\circ} 00^{\prime} 23^{\prime \prime}$ S; $72^{\circ} 08^{\prime} 34^{\prime \prime}$ ) $)$, entre los 100 a 280 m s.n.m. Son suelos profundos, que presentan una textura franco arcillosa en superficie y arcilloso en profundidad. Se caracterizan por presentar un buen drenaje y por ubicarse en sectores de pendiente compleja de hasta un 30\% (CIREN, 2003).

Se recolectaron muestras no disturbadas, en cilindros metálicos de $230 \mathrm{~cm}^{3}(7,2 \mathrm{~cm}$ de diámetro y $5,6 \mathrm{~cm}$ de alto) a $10 \mathrm{~cm}$ de profundidad. Además, se recolectaron agregados de suelo y muestras disturbadas en bolsas plásticas. Luego de la recolección del material, este fue sellado con film plástico y guardado en cajas para evitar pérdida de agua y daño mecánico.

Los lodos de salmón se obtuvieron del Departamento de Medio Ambiente de la empresa Multiexport, correspondientes al centro Puerto Fonck. Este centro trabaja con una tecnología de recirculación en la producción de alevines, con biofiltros de baja carga, construido por un estanque de concreto en el suelo que contiene un sustrato artificial que favorece la proliferación de bacterias que transforman el amonio. El contenido de MO de las muestras de lodo de salmón es de 35,7\%, duplicando el contenido presente en el horizonte superficial (Ap) de la Serie Los Ulmos (14\%). 


\section{Preparación de las muestras de suelo y lodo-suelo}

Las muestras disturbadas, fueron tamizadas a $2 \mathrm{~mm}$ y luego mezcladas con dos tratamientos de lodos (40 y $80 \mathrm{Mg} \mathrm{ha}^{-1}$ ). La mezcla fue ensamblada en cilindros de $375 \mathrm{~cm}^{3}$ a una densidad aparente de $0,7 \mathrm{~g} \mathrm{~cm}^{-3}$, con 7 repeticiones por tratamiento. Se aplicó el Test de Proctor para determinar una humectación óptima de las mezclas que permita una adecuada lubricación de las partículas (aproximadamente un 20\% de agua) para alcanzar la máxima densidad aparente (Dorner et al., 2011). Una vez terminado el ensamblaje se realizó una medición de permeabilidad de aire $(K a)$, según se detalla más adelante, para verificar la homogeneidad de las muestras.

Las muestras ensambladas se sometieron a ciclos de humectación y secado por medio de ascenso capilar y drenaje en bandejas de arena (60 hPa de tensión), respectivamente. Cada ciclo duró 7 días, con 2 días para la humectación $(0 \mathrm{hPa})$ y 5 para el drenaje $(-60 \mathrm{hPa})$. En total se efectuaron seis ciclos de humectación y secado. En cada ciclo se registraron los cambios volumétricos del suelo y la permeabilidad de aire $(K a)$.

\section{Análisis físicos}

Las muestras de suelo fueron caracterizadas mediante la determinación de la textura (Day, 1965; Forsythe, 1975), materia orgánica (Sadzawka et al., 2006) y densidad aparente por el método del cilindro (Sandoval et al., 2009).

La estabilidad de los agregados se midió por el método del tamizaje en seco y tamizaje en húmedo, usando un set de tamices de 20-12,5-8,0-6,3-4,0-1,0-0,5 mm, el que se agitó a una intensidad constante de $60 \mathrm{rpm}$ por 10 minutos. La variación del diámetro medio ponderado que experimentan los agregados (VDMA) entre los dos tamizajes indica el grado de estabilidad de estos (Hartge y Horn, 2009). Cada análisis se realizó con 3 repeticiones. La VDMA, se obtiene de la siguiente fórmula:

$$
\operatorname{VDMA}=\frac{\sum\left(n_{i 1} \cdot d_{i}\right)-\left(n_{i 2} \cdot d_{i}\right)}{\sum n_{i 1}}
$$

Donde, $\quad$ VDMA = variación del diámetro medio de los agregados $(\mathrm{mm})$ y para cada rango de tamaño $i$ de los tamices, $n_{i 1}=$ fracción tamizado en seco (\%); $n_{i 2}=$ fracción tamizado en húmedo (\%); $d_{i}=$ diámetro ponderado de los agregados (mm).

En cada ciclo de humectación y secado se registró el peso de la muestra (por medio de una balanza de precisión) y los cambios en la altura de ellas. Esto último se realizó con un profundímetro y un cilindro de referencia. El registro de los cambios volumétricos permitió determinar el índice de vacíos (Peng et al., 2007).
A partir del registro de los cambios en la altura de las muestras durante los ciclos, se procedió a determinar el coeficiente de extensibilidad lineal (COLE) que permite cuantificar la magnitud de la contracción (Peng et al., 2007) de acuerdo a la siguiente ecuación:

$$
\operatorname{COLE}=\frac{L_{0}-L_{-60}}{L_{105^{\circ} \mathrm{C}}}
$$

Donde, COLE $=$ Coeficiente de extensibilidad lineal (-); $L_{0}, L_{-60}, L_{105^{\circ} \mathrm{C}}=$ altura de la muestra a tensiones de $0 \mathrm{y}$ $60 \mathrm{hPa}$ y después de secar en el horno a $105^{\circ} \mathrm{C}$.

La conductividad de aire $(K I)$ se determinó al potencial mátrico de - $60 \mathrm{hPa}$ por medio del método de flujo constante en un permeámetro de aire, aplicando una diferencia de presión menor a $1 \mathrm{hPa}$, permitiendo un flujo laminar ascendente de aire a través de la muestra de suelo hasta alcanzar un flujo constante (Dörner y Dec, 2007). De esta manera, $K l$ se determinó a través de la siguiente ecuación:

$$
K l=\rho l * g *\left(\frac{\Delta V * L}{\Delta t * \Delta p * A}\right)
$$

Donde $K l=$ conductividad de aire $\left(\mathrm{m} \mathrm{s}^{-1}\right) ; \rho l=$ densidad de aire $\left(\mathrm{kg} \mathrm{m}^{-3}\right) ; g=$ aceleración de gravedad $\left(\mathrm{m} \mathrm{s}^{-2}\right) ; \Delta V$ = volumen de aire que fluye $\left(\mathrm{m}^{3}\right) ; L=$ largo del cilindro (m); $\Delta t=$ tiempo (s); $\Delta p=$ diferencia de presión (hPa); $A=$ área del cilindro $\left(\mathrm{m}^{2}\right)$. Finalmente, la conductividad de aire $(K I)$ se expresó en términos de permeabilidad de aire, mediante la siguiente ecuación:

$$
K a=K l * \frac{\eta}{\rho_{l} * g}
$$

donde $K a=$ permeabilidad de aire $\left(\mu \mathrm{m}^{2}\right) ; K l=$ conductividad de aire $\left(\mathrm{cm} \mathrm{s}^{-1}\right) ; \eta=$ viscosidad de aire $\left(\mathrm{g} \mathrm{s}^{-1} \mathrm{~cm}^{-1}\right)$; $\rho l=$ densidad de aire $\left(\mathrm{kg} \mathrm{m}^{-3}\right) ; g=$ aceleración de gravedad $\left(\mathrm{m} \mathrm{s}^{-2}\right)$. Las mediciones de la permeabilidad de aire se realizaron después de ser equilibradas las muestras a $60 \mathrm{hPa}$ en cada uno de los ciclos.

Una vez concluidos los ciclos, las muestras fueron saturadas por ascenso capilar para determinar la curva de retención de agua (curva pF). Luego, las muestras de suelo fueron equilibradas a tensiones de agua de $10,20,30,60,330$ y $15.430 \mathrm{~cm}$ de columna de agua. A partir de la curva de retención de agua se determinó la distribución de los poros del suelo (Hartge y Horn, 2009). Antes de secar las muestras a $105^{\circ} \mathrm{C}$, se recolectaron $10 \mathrm{~g}$ de suelo desde la parte superior e inferior de los cilindros de cada tratamiento para determinar el ángulo de contacto mediante una lupa de campo de visión horizontal (Ellies et al., 1995). Una vez determinada la curva $\mathrm{pF}$ y de contracción, se midió el ángulo 
de humectación o de contacto $(\alpha)$ que expresa la relación existente entre tensiones superficiales del sólido con el líquido, sólido con el gas y el líquido con el gas. Esta medición se realizó tanto para la superficie como también para la parte inferior de las muestras, debido a la proliferación de microorganismos en la superficie lo cual podría modificar las condiciones de evaluación.

\section{Análisis estadístico}

Como parte de la caracterización del suelo, se determinaron estadísticos descriptivos (media, mínimo y máximo estadístico, desviación estándar y varianza) de los parámetros evaluados. Además, se realizaron análisis de varianza factorial (dosis de lodo y ciclos de humectación y secado) y varianza de un factor (para el ángulo de contacto) los parámetros y tratamientos usados. Para las pruebas Post Hoc, se usó Tukey con un intervalo de confianza de 95\%.

\section{RESULTADOS}

\section{Propiedades estructurales de la Serie Los Ulmos}

La estabilidad de la estructura frente a la acción del agua se analizó por medio de la variación del diámetro medio ponderado de los agregados (VDMA). En la Figura 1 se aprecian las curvas de acumulación de agregados después del tamizaje en seco y en húmedo en la muestra de suelo original. La VDMA es de $0,28 \mathrm{~mm}$.

Además, la VDMA permite determinar el efecto de la aplicación de lodos sobre la estabilidad de los agregados, donde se observó una reducción de este pará- metro con un incremento en la dosis de lodo de salmón (Cuadro 1), la que fue estadísticamente significativa al comparar la dosis de $80 \mathrm{Mg} \mathrm{ha}^{-1}$ con material recolectado en terreno $(\mathrm{p}<0,05)$.

En el suelo evaluado, predominan los poros de agua útil, con una media de $30,2 \%$, seguido por los poros de agua inútil $(19,8 \%)$, los poros de drenaje rápido $(14,5 \%)$ y por último los poros de drenaje lento, con una media de $7,3 \%$ (Cuadro 1 ).

\section{Efecto de la aplicación de diferentes dosis de lodos de salmón sobre las propiedades físicas del suelo Los Ulmos}

La secuencia de ciclos de mojado y secado implica aproximadamente un $20 \%$ de cambio en el contenido de agua base suelo húmedo en cada uno de los ciclos (Figura 2). El cambio en el contenido de agua durante los ciclos induce variaciones en el volumen del suelo (Figura 3), donde la contracción del suelo es mayor que la capacidad de este para recuperarse durante la humectación. Si bien las variaciones del contenido de agua durante los ciclos de mojado y secado no mostraron diferencias entre tratamientos, la relación de vacíos en general fue menor en el Testigo durante el proceso de estabilización estructural producto de estos ciclos.

La magnitud de la contracción se evaluó a través del COLE, que define la variación del volumen del suelo en una dimensión (vertical) en la medida que disminuye el contenido de agua (Figura 4). Los valores de COLE reflejan que el movimiento de las partículas sólidas y su reordenamiento durante los ciclos de mojado y secado producen cambios en la porosidad de aire (Figura 5).

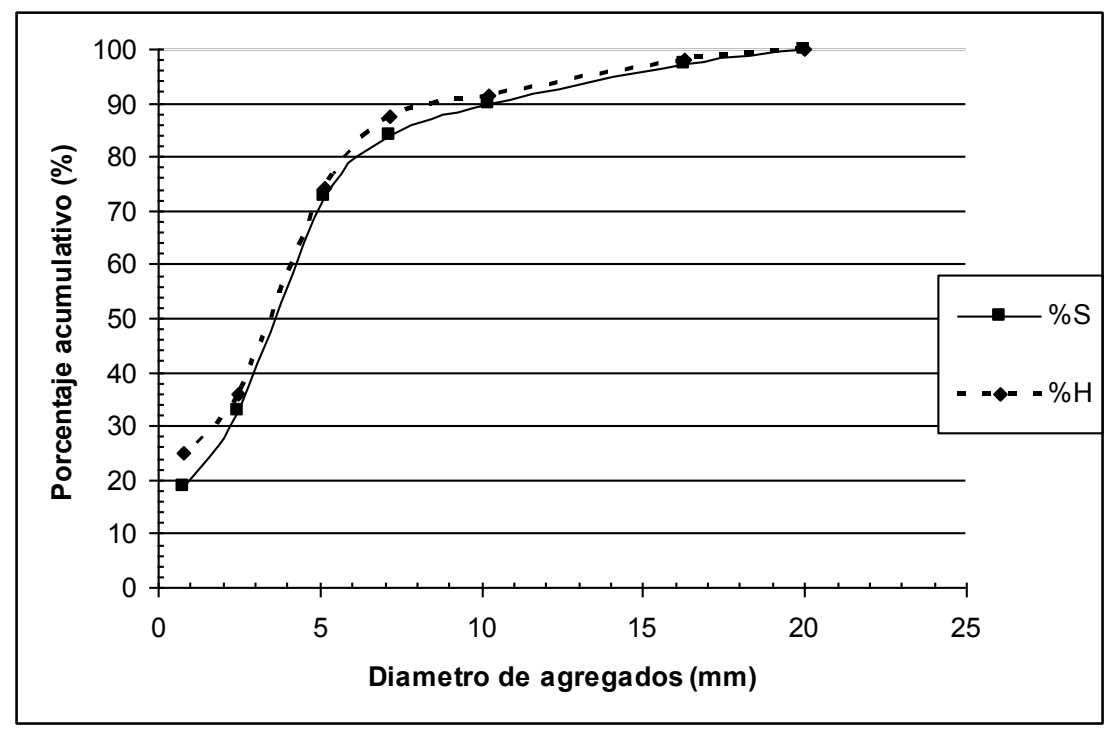

Figura 1. Distribución acumulada de los agregados del suelo. $\mathrm{S}=$ tamizado en seco y $\mathrm{H}=$ tamizado en húmedo.

Figure 1. Accumulated distribution of soil aggregates. $\mathrm{S}=$ dry sieving and $\mathrm{H}=$ wet sieving. 
La permeabilidad de aire no muestra una tendencia clara a través de los ciclos de humectación y secado, sin embargo, existieron diferencias significativas $(p<0,05)$ entre el testigo y los tratamientos de 40 y $80 \mathrm{Mg} \mathrm{ha}^{-1}$ (Figura 6). Además, la aplicación de lodo tuvo un efecto significativo $(\mathrm{p}<0,05)$ sobre los poros de drenaje rápido, lento y de agua útil entre los tratamientos y con respecto a la condición del suelo original (Cuadro 1).

Para el caso de los poros de drenaje rápido (PDR), el tratamiento de $80 \mathrm{Mg} \mathrm{ha}^{-1}$ de lodos es el que presenta una menor porosidad, seguido por el tratamiento de
$40 \mathrm{Mg} \mathrm{ha}^{-1}$, testigo y el material estructurado. Los poros de drenaje lento (PDL) no mostraron diferencias significativas $(p>0,05)$ entre las diferentes dosis de aplicación, pero sí con respecto al material estructurado, el cual fue menor. En cuanto a la capacidad de almacenamiento de agua aprovechable, el material estructurado presenta el mayor porcentaje de poros de agua útil (PAU); como consecuencia de los tratamientos, se puede observar que al aplicar $80 \mathrm{Mg} \mathrm{ha}^{-1}$ de lodo aumentaron los poros de agua útil en compensación a la pérdida de poros de drenaje rápido y lento.

Cuadro 1. Variación del diámetro medio de los agregados, ángulo de contacto y distribución de poros para los diferentes tratamientos.

Table 1. Variation of mean aggregate diameter, contact angle and pore-size distribution for the different treatments.

\begin{tabular}{|c|c|c|c|c|c|c|c|}
\hline \multirow{2}{*}{ Tratamientos } & \multirow{2}{*}{$\begin{array}{l}\text { VDMA } \\
(\mathrm{mm})\end{array}$} & \multicolumn{2}{|c|}{ Angulo de humectación $\left({ }^{\circ}\right)^{*}$} & \multirow{2}{*}{ PDR } & \multirow{2}{*}{ PDL } & \multirow{2}{*}{ PAU } & \multirow{2}{*}{ PAI } \\
\hline & & Área superior & Área Inferior & & & & \\
\hline $80 \mathrm{Mg} \mathrm{ha}^{-1}$ & $0,12 \pm 0,09 \mathrm{a}$ & $31,7 \pm 1,63 \mathrm{a}$ & $36,4 \pm 1,74 b$ & $6,1 \pm 0,70 b$ & $17,9 \pm 0,68 \mathrm{a}$ & $23,6 \pm 0,52 b$ & $19,6 \pm 0,37 \mathrm{a}$ \\
\hline $40 \mathrm{Mg} \mathrm{ha}^{-1}$ & $0,17 \pm 0,02 \mathrm{ab}$ & $25,8 \pm 1,01 \mathrm{c}$ & $24,9 \pm 1,01 \mathrm{c}$ & $9,9 \pm 1,72 \mathrm{ab}$ & $18,3 \pm 0,62 \mathrm{a}$ & $21,2 \pm 0,7 \mathrm{bc}$ & $18,9 \pm 0,36 \mathrm{a}$ \\
\hline Testigo & $0,21 \pm 0,02 \mathrm{ab}$ & $17,4 \pm 0,78 \mathrm{~d}$ & $16,5 \pm 0,57 \mathrm{~d}$ & $11,4 \pm 1,25 \mathrm{a}$ & $16,5 \pm 0,48 a$ & $20,6 \pm 0,67 c$ & $19,3 \pm 0,37 \mathrm{a}$ \\
\hline Muestras de Terreno & $0,28 \pm 0,06 \mathrm{~b}$ & - & - & $14,5 \pm 1,15 \mathrm{a}$ & $7,3 \pm 0,44 b$ & $30,2 \pm 0,69 a$ & $19,8 \pm 0,38 a$ \\
\hline
\end{tabular}

VDMA: variación del diámetro medio de los agregados, PDR: poros de drenaje rápido; PDL: poros de drenaje lento; PAU: poros de agua útil; PAI: poros de agua inútil, distribución del tamaño de poros determinado al finalizar los ciclos. Letras distintas indican diferencias significativas (Tukey HSD 95\%) en la columna de VDMA y distribución de tamaño de poros. *Para ángulo de humectación determinado al finalizar los ciclos, las letras distintas indican diferencias significativas para cada tratamiento y área de medición (Tukey HSD 95\%). Valores promedios con error estándar, $\mathrm{n}=3$.

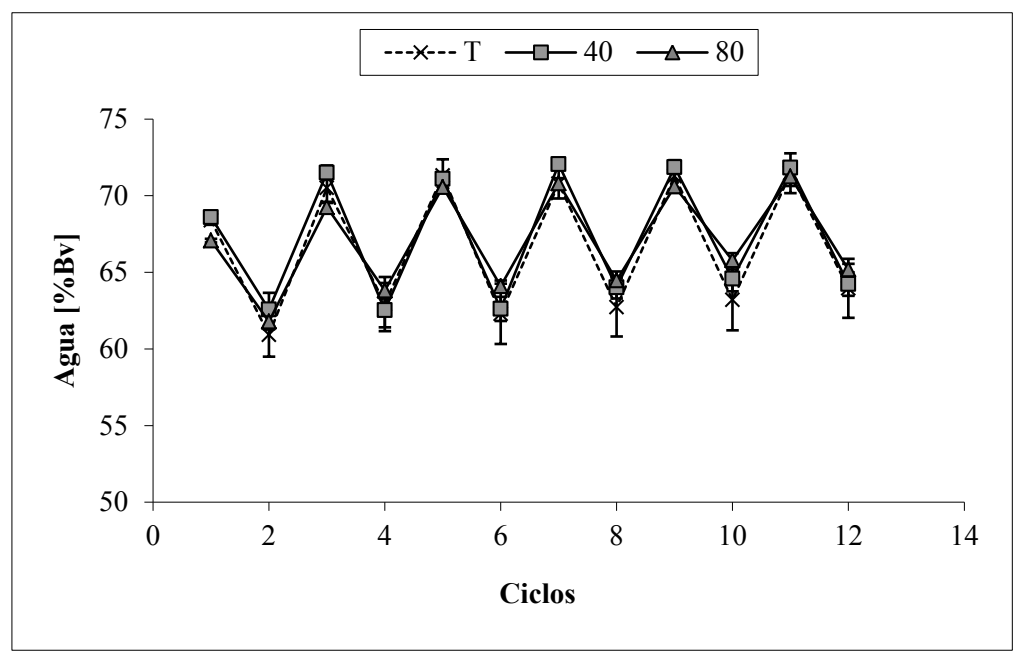

Figura 2. Cambios del contenido de agua del suelo a través de los ciclos de humectación y secado. T = muestras testigo, 40 Ton y 80 Ton = cantidad de lodos en Mg agregado por ha ${ }^{-1}$. La primera medición corresponde a muestras saturadas de un total de 12 mediciones ( $\mathrm{n}=7 \mathrm{y}$ las barras indican el error estándar).

Figure 2. Changes in soil water content through the wetting and drying cycles. $\mathrm{T}=$ control samples, 40 Ton and 80 Ton $=$ amount of sluge in Mg added per ha ${ }^{-1}$. The first measurement corresponds to saturated samples out of a total of 12 measurements $(\mathrm{n}=$ 7; the bars indicate the standard error). 
El ángulo de contacto permite determinar el nivel de hidrofobia del suelo frente a la aplicación de lodos de salmón. Se observó un incremento estadísticamente significativo $(\mathrm{p}<0,05)$ en el ángulo de contacto con dosis crecientes de lodo (Cuadro 1). Para el caso del tratamiento de $80 \mathrm{Mg} \mathrm{ha}^{-1}$, también se observaron diferencias significativas entre el área superior e inferior del cilindro.

\section{DISCUSIÓN}

Efecto de la aplicación de lodos sobre las propiedades físicas del suelo después de los ciclos de humectación y secado

Seguel y Horn (2006) señalan que la generación de estructura es el resultado de los ciclos de humectación

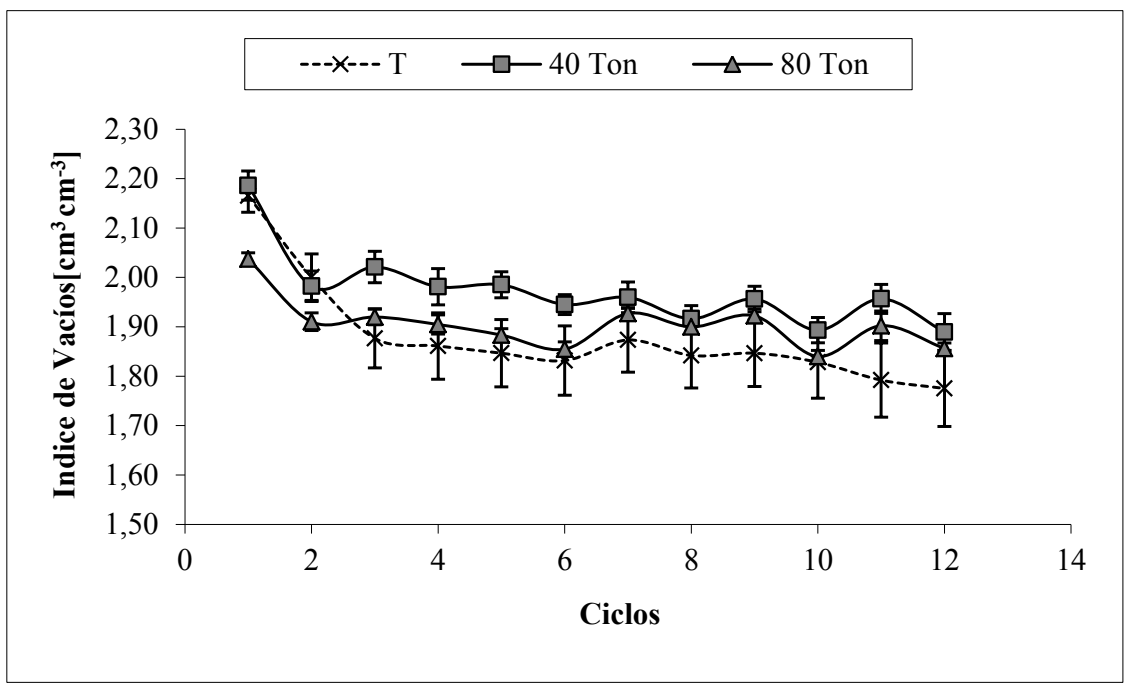

Figura 3. Cambios en el volumen del suelo a través de los ciclos de humectación y secado. T = muestras testigo, 40 Ton y 80 Ton = cantidad de lodos en Mg agregado por ha ${ }^{-1}$. La primera medición corresponde a muestras saturadas de un total de 12 mediciones ( $\mathrm{n}=7 \mathrm{y}$ las barras indican el error estándar).

Figure 3. Changes in soil volume through the wetting and drying cycles. $\mathrm{T}=$ control samples, 40 Ton and 80 Ton $=$ amount of sluge in Mg added per ha-1. The first measurement corresponds to saturated samples out of a total of 12 measurements $(\mathrm{n}=7$; the bars indicate the standard error).

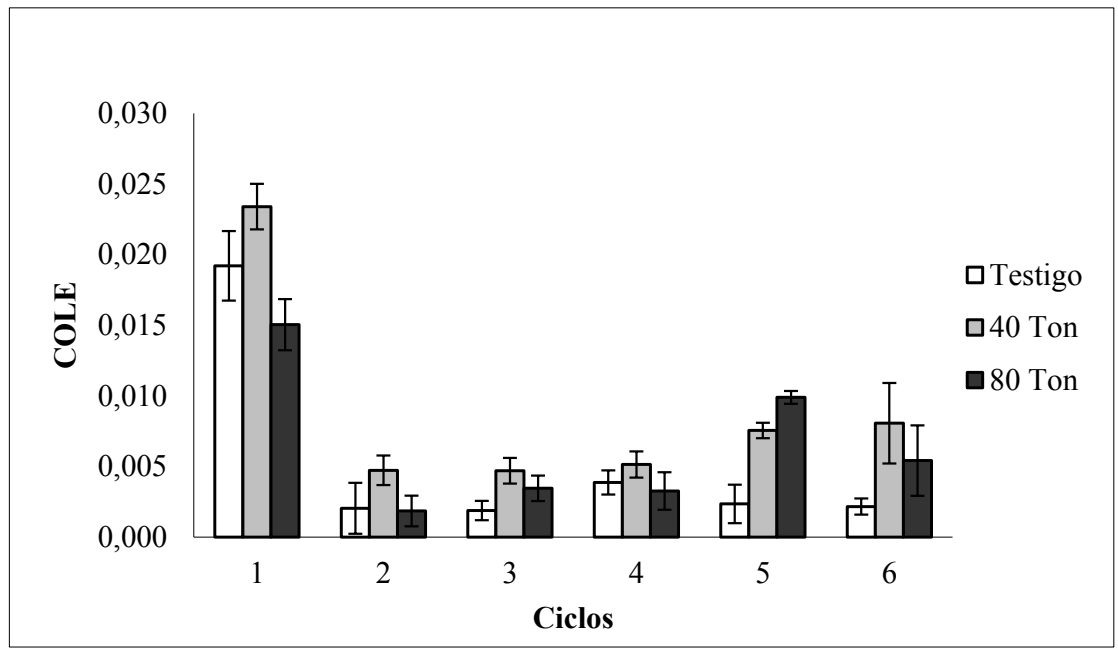

Figura 4. Cambios en el coeficiente de extensibilidad lineal (COLE) durante los ciclos.

Figure 4. Changes in the coefficient of linear extensibility (COLE) during cycles. 
y secado (para un Andisol tamizado), donde la contracción inicial resulta en un decrecimiento irreversible del espacio poroso y en un incremento en la densidad aparente. Lo anterior coincide con los resultados, que muestran diferencias significativas entre los primeros dos ciclos y los siguientes, siendo la contracción inicial la más notoria (Figura 3), lo que se ve reflejado en el coeficiente de extensibilidad lineal (COLE, Figura 4). La contracción observada en todos los tratamientos implica un reordenamiento de las partículas que se manifiesta en una reducción de la porosidad de aire y aumento de la densidad aparente (Horn y Smucker, 2005), debido principalmente a que las partículas de materia orgánica asociadas a los lodos ocupan el espa-

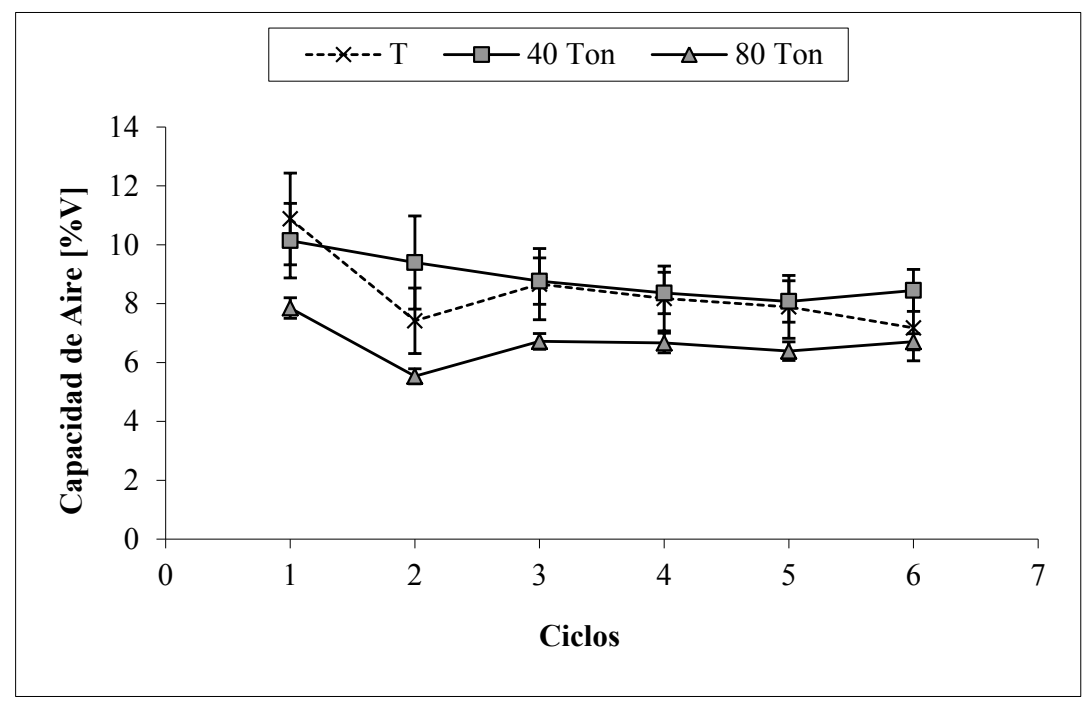

Figura 5. Capacidad de aire a través de los ciclos de humectación y secado. $\mathrm{T}=$ muestras testigo, 40 Ton y 80 Ton $=$ cantidad de lodos en Mg agregado por ha ${ }^{-1}$ ( $\mathrm{n}=7$; las barras indican el error estándar).

Figure 5. Air capacity during the wetting and drying cycles. $\mathrm{T}=$ control samples, 40 Ton and $80 \mathrm{Ton}=$ amount of sluge in $\mathrm{Mg}$ added per ha-1 $(n=7$; the bars indicate the standard error).

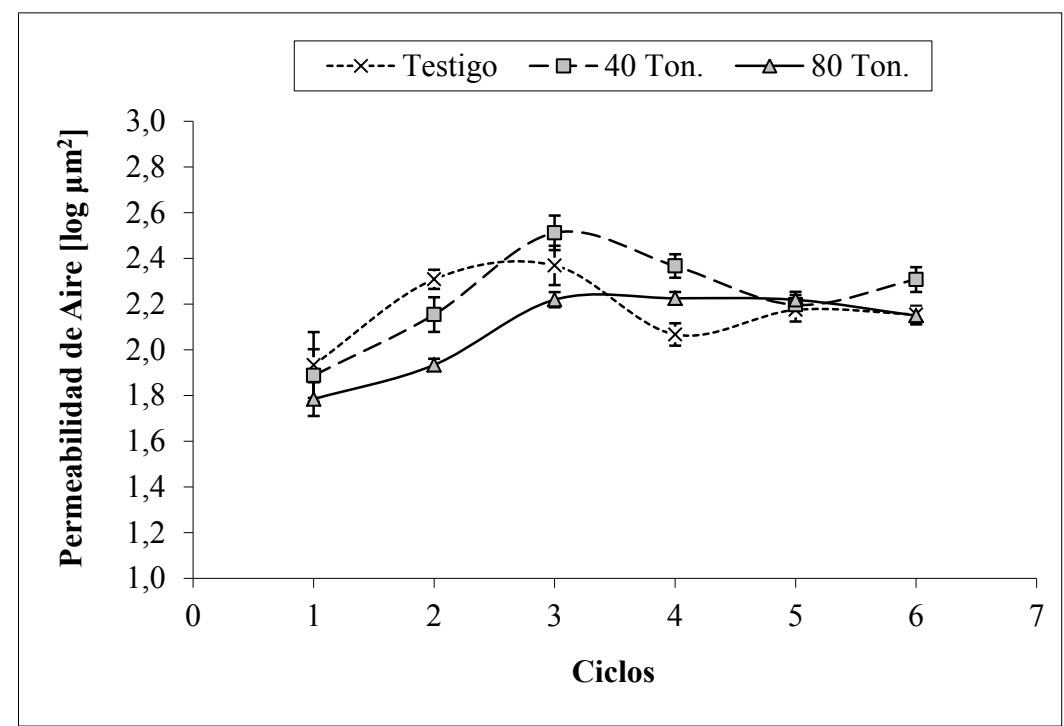

Figura 6. Dinámica en la permeabilidad de aire del suelo a través de los ciclos de humectación y secado. Las barras indican error estándar $(\mathrm{n}=3)$.

Figure 6. Dynamics in the air permeability of the soil through the wetting and drying cycles. The bars indicate the standard error $(n=3)$. 
cio entre los agregados del suelo. En el tratamiento de $80 \mathrm{Mg} \mathrm{ha}^{-1}$ de aplicación de lodo de salmón se observó una disminución de los poros de drenaje rápido, la cual es compensada con un aumento de los poros de agua útil (Cuadro 1). La sustitución de la macroporosidad por poros más finos en el suelo debe considerarse como una modificación de la estructura del suelo (Ellies et al., 1993); en este caso, la redistribución de poros gruesos a medianos, como consecuencia del espacio ocupado por las partículas de MO entre los agregados de suelo, equivale a un incremento en la capacidad de almacenamiento de agua útil, pero a expensas de una menor aireación e infiltración del agua (Seguel y Horn, 2006).

Los ciclos de humectación y secado reordenan las partículas y agregados del suelo, afectando la distribución de poros y las vías de flujo. En ese contexto, las mediciones de $\mathrm{Ka}$ en los ciclos ratifican que existen variaciones en la estructura, donde se observaron cambios en la fracción de poros que conducen aire (Figura 5) y su continuidad. Tuli et al. (2005) mostraron que la permeabilidad de agua y aire fue menor en muestras disturbadas que en muestras no disturbadas (con estructura), especialmente la permeabilidad de aire, debido a su mayor dependencia con respecto a la estructura y agregados del suelo y a su sensibilidad como indicador de funcionalidad del sistema poroso (Horn y Fleige, 2009). Por su parte, Salazar et al. (2012) encontraron un aumento en la infiltración de agua con aplicaciones de lodos urbanos, con diferencias significativas respecto al testigo en dosis mayores a $60 \mathrm{Mg} \mathrm{ha}^{-1}$. En el presente estudio, si bien es cierto que no se observó un efecto concluyente de la dosis de aplicación de lodos sobre $K a$, sí es claro que los ciclos de humectación y secado permiten la formación de un sistema poroso que permite una mayor conducción de aire, respecto a la situación original.

Por otro lado, un incremento en la aplicación de lodo de salmón, y por lo tanto, en el contenido de MO, aumenta la estabilidad estructural del suelo frente a la acción del agua. Esto coincide con lo planteado por Ellies et al. (1996), quienes determinaron que en suelos bajo bosque o con un alto contenido de MO, se caracterizan por un menor diámetro medio de los agregados, presentando una mayor proporción de agregados estables con un diámetro $<2 \mathrm{~mm}$. Un incremento en el contenido de MO de un suelo produce un aumento en la resistencia a la humectación, tal que se produce una disminución en la variación del diámetro medio de los agregados (Ellies et al., 2002). Esto es consistente con los observado en el presente estudio, debido al efecto cementante de la MO y al incremento en la resistencia a la humectación, lo que permite la difusión de aire sin que se produzca una destrucción del agregado por el rápido ingreso del agua al interior del agregado (Hartge y Horn, 2009). Es así, que sitios que presentan un suelo hidrófobo presentan agregados estables a la dis- persión y por ende exhiben una pequeña variación del diámetro medio de los agregados después de un tamizado en seco y húmedo.

La estabilidad de los agregados del suelo mejora y se mantiene con una mayor proporción de componentes hidrofóbicos en la MO respecto a componentes hidrofílicos (Piccolo y Mbagwu, 1999). La adición de biosólidos aumenta la cantidad de MO y con ello se produce una mayor actividad microbiana (Salazar et al., 2012), cuyos productos secundarios como consecuencia de la respiración biológica generan una mayor repelencia al agua y una mayor estabilidad estructural (Savage et al., 1969; Hallett y Young, 1999).

Finalmente, cabe destacar que una moderada hidrofobia del suelo superficial es deseable (Ellies et al., 1995). La razón práctica es que un suelo hidrófobo, con agregados estables al agua, no forma costras cuando está descubierto, ya que se previene la dispersión de partículas, reduciéndose con ello la escorrentía superficial y el arrastre de sedimentos (Lado et al., 2004). Sin embargo, una hidrofobia excesiva favorece al escurrimiento superficial y el arrastre de material agregado, afectando también la disponibilidad del agua para las plantas (Hallet et al., 2001).

\section{CONCLUSIONES}

Con el tratamiento de $80 \mathrm{Mg} \mathrm{ha}^{-1}$ de aplicación de lodo de salmón se observó una sustitución de la macroporosidad por otra que permite almacenar agua aprovechable para las plantas. Con el incremento en el contenido de lodo, disminuye la VDMA y aumenta la repelencia al agua, permitiendo que el suelo sea más estable a la acción dispersante del agua. Sin embargo, la capacidad de flujo de aire no se vio alterada por los tratamientos.

El reciclaje de lodo de salmón se presenta como una alternativa viable de ser realizada en suelos degradados. Sin embargo, es necesario realizar mayores estudios, muchos de ellos a largo plazo, como evaluar sucesivas dosis en un mismo sitio (p.ej. analizar efectos sobre la repelencia al agua) y también la forma de aplicación de los residuos orgánicos.

\section{REFERENCIAS}

Ahmed, H.K., Fawy, H.A., Abdel-Hady, E.S., 2010. Study of sewage sludge use in agriculture and its effect on plant and soil. Agriculture and Biology Journal of North America 1(5), 1044-1049.

Aravena, C., Valentin, C., Diez, C., Mora, M., Gallardo, F., 2007. Aplicación de lodos de planta de tratamiento de celulosa: efecto en algunas propiedades físicas y químicas de suelos volcánicos. Revista de la Ciencia del Suelo y Nutrición Vegetal 7(1), 1-14.

Baldock, J.A., Nelson, P.N., 1999. Soil organic matter, in: Sumner, M.E. (Ed.), Handbook of Soil Science. CRC Press, 
Boca Raton, pp. B25-B71.

Celis, J., Sandoval, M., Zagal, E., Briones, M., 2006. Efecto de la adición de biosolidos urbanos y de salmonicultura sobre la germinación de semillas de lechuga (Lactuca sativa L.) en un suelo patagónico. Revista de la Ciencia del Suelo y Nutrición Vegetal 6(3), 13-25.

Celis, J., Sandoval, M., Barra, R., 2008. Plant response to salmon wastes and sewage sludge used as organic fertilizer on two degraded soils under greenhouse conditions. Chilean Journal of Agricultural Research 68(3), 247-283.

Celis, J., Sandoval, M., 2010. Agricultural potential of salmon wastes used as organic fertilizer on two Chilean degraded soils. $19^{\text {th }}$ World Congress of Soil Science, Soil Solutions for a Changing World 1-6 August 2010, Brisbane, Australia, pp. 17-20. https://iuss.org/19th\%20WCSS/ Symposium/pdf/0574.pdf

Centro de Información de Recursos Naturales (CIREN), 2003. Estudio Agrológico X Región. Descripciones de Suelos Materiales y Símbolos. Publicación CIREN N 123 , Santiago, Chile.

Cuevas, J., Seguel, O., Ellies, A., Dörner, J., 2006. Efectos de las enmiendas orgánicas sobre las propiedades físicas del suelo con especial referencias a la adición de lodos urbanos. Revista de la Ciencia del Suelo y Nutrición Vegetal 6(2), 1-16.

Day, P. R., 1965. Particle fractionation and particle-size analysis, in: Black, C.A. (Ed.), Methods of Soil Analysis, Part I. Agronomy 9, ASA and SSSA. Madison, Wisconsin, pp. 545-567.

Diacono, M., Montemurro, F., 2011. Long-term effects of organic amendments on soil fertility, in: Lichtfouse E., Hamelin, M., Navarrete, M., Debaeke, P. (Eds.), Sustainable Agriculture Volume 2. Springer, Dordrecht, pp. 761-786.

Dörner, J., Dec, D., 2007. La permeabilidad del aire y conductividad hidráulica saturada como herramienta para la caracterización funcional de los poros del suelo Revista de la Ciencia del Suelo y Nutrición Vegetal 7(2), 1-13.

Dörner, J., Sandoval, P., Dec, D., 2010. The role of soil structure on the pore functionality of an Ultisol. Journal of Soil Science and Plant Nutrition 10(4), 495-508.

Ellies, A., Ramírez, C., Figueroa, H., 1993. Modificaciones estructurales de un suelo sometido a distintos usos forestales. Bosque 14(2), 25-30.

Ellies, A., Grez, R., Ramírez, C., 1995. Potencial de humectación y estabilidad estructural de suelos sometidos a diferentes manejos. Agricultura Técnica 55(3-4), 220-225.

Ellies, A., Grez, R., Ramírez, C., 1996. Efecto de la materia orgánica sobre la capacidad de humectación y las propiedades estructurales de algunos suelos de la zona centro sur de Chile. Agro Sur 24(1), 48-58.

Ellies, A., Mac Donald, R., Ramírez, C., 2002. Efecto de la resistencia a la humectación sobre la estabilidad al agua de los agregados del suelo. Revista de la Ciencia del Suelo y Nutrición Vegetal 2(2), 1-9.

Forsythe, W., 1975. Manual de laboratorio de física de suelos. Instituto Interamericano de Ciencias Agrícolas de la OEA. Turrialba, Costa Rica.

Guerrero, C., Mataix-Solera, J., Navarro-Pedreño, J., GarcíaOrenes, F., Gómez, I., 2001. Different patterns of aggregate stability in Burned and restored Soils. Arid Land Research and Management 15(2), 163-171.
Hallet, P., Baumgartl, T., Young, I., 2001. Subcritical water repellency of aggregates from a range of soil management practices. Soil Science Society of America Journal 65, 184-190.

Hallet, P., Young, M., 1999. Changes to water repellence of soil aggregates caused by substrate-induced microbial activity. European Journal of Soil Science 50, 35-40.

Hartge, K., Horn, R., 2009. Die physikalische Untersuchung von Böden. Praxis, Messmethoden, Auswertung. E. Schweizerbart'sche Verlagsbuchhandlung. Stuttgart. $178 \mathrm{p}$.

Hillel, D., 1998. Environmental Soil Physics. Academic Press, San Diego.

Horn, R., 2002. Soil mechanical properties and processes in structured unsaturated soils under various landuse and management systems, in: Pagliai, M., Jones, R. (Eds.), Sustainable Land Management-Environmental Protection. A Soil Physical Approach. Advances in Geoecology, vol. 35. Catena Publ. Reiskirchen, Germany, pp. 305-318.

Horn, R., Smucker, A., 2005. Structure formation and its consequences for gas and water transport in unsaturated arable and forest soil. Soil and Tillage Research 82, 5-14.

Horn, R., Fleige, H., 2009. Risk assessment of subsoil compaction for arable soils in Northwest Germany at farm scale. Soil and Tillage Research 102, 201-208.

Lado, M., Paz, A., Ben-Hur, M., 2004. Organic matter and aggregate size interactions in infiltration, seal formation, and soil loss. Soil Science Society of America Journal 68, 935-942.

Mena, G., 1997. Evaluación experimental de la capacidad de Diplodon chilensis para procesar los excedentes orgánicos generados por la salmonicultura. Tesis Lic. Biología Marina. Valdivia. Universidad Austral de Chile, Facultad de Ciencias. 60 p.

Peng, X., Horn, R., Smucker, A., 2007. Pore shrinkage dependency of inorganic and organic soils on wetting and drying cycles. Soil Science Society of America Journal 71(4), 1095-1104.

Peralta, M., 1995. Conservación y degradación de suelos en Chile. Santiago, Chile.

Pinochet, D., Artacho, P., Azúa, P., 2001. Potencialidad como abono orgánico de los desechos sólidos subproductos del cultivo de peces Salmonideas. Agro Sur 29(1), 78-82.

Piccolo, A., Mbagwu, J., 1999. Role of hydrophobic components of soil organic matter in soil aggregate stability. Soil Science Society of America Journal 63, 1801-1810.

Sadzawka, A., Carrasco, M., Grez, R., Mora, M., Flores, H., Neaman, A., 2006. Métodos de análisis recomendados para los suelos de Chile. Centro de Investigación Regional La Platina. Serie Actas INIA 34 (1). Santiago, Chile.

Salazar, I., Millar, D., Lara, V., Núñez, M., Parada, M., Alvear, M., Barahona, J., 2012. Effects of the application of biosolids on some chemical, biological and physical properties in an Andisol from southern Chile. Journal of Soil Science and Plant Nutrition 12(3), 441-450.

Sandoval, P., 2009. Curva de retención de humedad, de contracción y de permeabilidad de aire en función de distintas densidades aparentes de un suelo rojo arcilloso. Tesis Ingeniero Agrónomo. Valdivia. Universidad Austral de Chile, Facultad de Ciencias Agrarias. 67 p.

Savage, S., Martin, J., Letey, J., 1969. Contribution of some soil 
fungi to natural and heat-induced water repellency in sand. Soil Science Society of America Journal 33, 405-409.

Seguel, O., Horn, R., 2006. Structure properties and pore dynamics in aggregate beds due to wetting-drying cycles. Journal of Plant Nutrition and Soil Science 169(2), 221232.

Teuber, N., Alfaro, M.A., Salazar, F.J., Bustos, C., 2005. Sea salmon sludge as fertilizer: effects on a volcanic soil and annual ryegrass yield and quality. Soil Use and Management 21(4), 432-434.
Tuli, A., Hopmans, J., Rolston, D., Moldrup, P., 2005. Comparison of air and water permeability between disturbed and undisturbed soil. Soil Science Society of America Journal 69(5), 1361-1371.

Varnero, M., 2001. Desarrollo de sustratos orgánicos: compost y bioabonos, in: Universidad de Chile, Facultad de Ciencias Agrarias y Forestales, Departamento de Manejo de Recursos Forestales (Ed.), Experiencias Internacionales en la Rehabilitación de Espacios Degradados, Proyecto FONDEF D98I1036. Santiago, Chile, pp. 21-30. 